\title{
Predictive Gait Quality Measures Using Modular Neuromuscular Control Parameters in Chronic Post- stroke Individuals
}

\section{Sung Yul Shin}

Shirley Ryan AbilityLab https://orcid.org/0000-0002-3210-8417

\section{Yusung Kim}

Korea Advanced Institute of Science and Technology

Arun Jayaraman

Shirley Ryan AbilityLab

Hyung-Soon Park ( $\nabla$ hyungspark@kaist.ac.kr)

https://orcid.org/0000-0003-4274-7420

\section{Research}

Keywords: muscle module, muscle synergy, gait quality, gait symmetry, stroke

Posted Date: July 17th, 2020

DOI: https://doi.org/10.21203/rs.3.rs-43589/v1

License: (c) (i) This work is licensed under a Creative Commons Attribution 4.0 International License. Read Full License 
Parameters in Chronic Post-Stroke Individuals

3

4 Sung Yul Shin, PhD

5 Department of Mechanical Engineering

6 Korea Advanced Institute of Science and Technology (KAIST)

7 Daehak-ro 291, Yuseong-gu, Daejeon 34141, Republic of Korea

8 Max Nader Lab for Rehabilitation Technologies and Outcomes Research

9 Shirley Ryan AbilityLab

10355 E Erie St, Chicago, IL, 60611, USA

11

12

13

\section{Yusung Kim, MS}

Department of Mechanical Engineering

Korea Advanced Institute of Science and Technology (KAIST)

Daehak-ro 291, Yuseong-gu, Daejeon 34141, Republic of Korea

\section{Arun Jayaraman, PT, PhD}

Max Nader Lab for Rehabilitation Technologies and Outcomes Research

Shirley Ryan AbilityLab

355 E Erie St, Chicago, IL, 60611, USA

Department of Physical Medicine and Rehabilitation

Northwestern University

710 N Lake Shore Dr, Chicago, IL, 60611, USA

\section{Hyung-Soon Park, PhD $^{1}$}

Department of Mechanical Engineering

Korea Advanced Institute of Science and Technology (KAIST)

Daehak-ro 291, Yuseong-gu, Daejeon 34141, Republic of Korea

hyungspark@kaist.ac.kr

\footnotetext{
${ }^{1}$ Corresponding author
} 


\section{Abstract}

33 Background: Recent evidence suggests that disinhibition and/or hyperexcitation of the

34 brainstem descending pathways and intraspinal motor network diffuse spastic synergistic

35 activation patterns after stroke. This results in simplified or merged muscle sets (i.e., muscle

36 modules or synergies) compared to non-impaired individuals and this leads to poor walking

37 performance. However, the causal relations of how these neuromuscular deficits influence gait

38 quality (e.g., symmetry or natural walking patterns) are still unclear. The objective of this

39 exploratory study was to investigate the relations of modular neuromuscular framework and gait

40 quality measures in chronic stroke individuals.

41 Methods: Sixteen chronic post-stroke individuals participated in this study. Full lower body

42 three-dimensional kinematics and electromyography (EMG) were concurrently measured during

43 overground walking at a comfortable speed. We classified subjects into two groups based on the

44 number of muscle modules and compared gait quality measures using a two-sampled t-test.

45 Then, a stepwise multiple regression was used to investigate the optimal combination of the

46 neuromuscular parameters to predict gait quality measures.

47 Results: Subjects who had a reduced number of muscle modules had greater asymmetry in the

48 kinematic parameters including limb length $(\mathrm{p}<0.01)$, footpath area $(\mathrm{p}<0.01)$, hip $(\mathrm{p}<0.05)$ and

49 knee $(\mathrm{p}<0.01)$ flexion/extensions, and hip abduction/adduction $(\mathrm{p}<0.01)$. We also found that the

50 gait quality measures were predictable with the input variables from the modular neuromuscular

51 control framework including variability accounted for $(V A F)$ information from the muscle

52 modules and area under the EMG envelope curves of the quadriceps (i.e., rectus femoris and

53 vastus lateralis) and tibialis anterior muscles with significant association (average $R^{2}=45.6 \%$ ). 
54 Conclusions: The results suggest that there exists a strong correlation between the

55 neuromuscular control framework and the gait quality measures. This study helps to understand

56 the underlying causality of disturbances in gait quality and provides insight for a more

57 comprehensive outcome measure to assess gait impairment after stroke.

58

59 Key words: muscle module, muscle synergy, gait quality, gait symmetry, stroke

60 


\section{Background}

Effective gait recovery after a stroke involves improvements both in functional mobility and quality of movement. Most of the clinical outcomes in gait studies have focused on functional indices that provide a holistic picture of walking performance and recovery [1], [2]. On the other hand, an increased number of recent studies accentuate the importance of monitoring detailed gait quality to assess gait impairments [3]. Disturbances in gait quality are associated with an increased risk of falls [4], greater energy expenditure [5], and long-term problems such as learned non-use or use-dependent plasticity, musculoskeletal injuries, and pain [6], [7].

Symmetry is a common measure to characterize disturbances in gait quality. While spatiotemporal symmetry (e.g., step length, step time) has been well charted to describe walking [8], post-stroke individuals exhibit significant asymmetry in joint kinematics with greater interindividual variability than spatiotemporal measures [9]. Typical asymmetry in joint kinematics includes reduced hip extension, knee flexion and ankle dorsi/plantarflexion, and knee hyperextension on the impaired side [10]. Limb kinematics, related to the end-effector (i.e., foot) motion in task space, has also been indicated as an important parameter for locomotor function [3]. For instance, Shin et al., found that post-stroke individuals preferentially coordinated the paretic side of limb function using limb kinematics by compensating joint kinematics during walking [3].

Neuromuscular activity is crucial to execute biomechanical functions such as gait [11]. Previous studies have shown that muscle activity during walking can be grouped into sets of coexcited muscles (also known as muscle modules or synergies) [12]. These modules may originate from neural-adaptations caused by repetitive activities and may reduce the computational cost in selecting strategies of motor coordination [13]. Previous studies have identified that well- 
84 coordinated gait in healthy individuals can be produced by four group of modules [12]. Other

85 studies suggest that the concept of muscle modules can be used as an outcome measure to assess

86 motor recovery following therapeutic interventions [14].

87 Recent evidence suggests that disinhibition and/or hyperexcitation of the brainstem

88 descending pathways and intraspinal motor network diffuse spastic synergistic activation post-

89 stroke [15]. As a result, simplified or merged muscle sets compared to non-impaired individuals

90 are typically observed and lead to poor walking performance, for instance, reduced walking

91 speed with greater spatiotemporal asymmetry than those of healthy individuals [12]. While this

92 study discusses somewhat the influence of neuromuscular deficit on gait impairment, no

93 evidence exists to show how detailed gait quality measures are influenced by neuromuscular

94 deficits. Thus, exploring relations between gait quality measures and neuromuscular deficits,

95 such as merged muscle modules, may help to explain the underlying causality among

96 impairments and locomotor functions after stroke.

97 The objective of this exploratory study was to investigate the relations of modular neuromuscular deficits and disturbances in gait quality measures (i.e., asymmetry) in terms of 99 spatiotemporal, limb and joint kinematic parameters in chronic post-stroke individuals (see Fig.

100 1). We measured lower body electromyography (EMG) activities and gait kinematics

101 concurrently during walking from 16 participants. We hypothesized that the post-stroke group

102 with a reduced number of muscle modules will exhibit greater asymmetry in gait quality

103 measures due to the loss of independence in motor activations [12]. Accordingly, we also

104 expected to predict the gait quality measures with the input modular neuromuscular control

105 framework assuming a causal relationship exists in these measures [16]. 


\section{Methods}

\section{Participants}

We recruited 16 individuals (6 left hemiparesis, 12 male, age: $62.9 \pm 11.1$ years) with chronic stroke (> 6 months) to participate in this study approved by the Institutional Review Board of Korea Advanced Institute of Science and Technology. Individuals ranged in age and impairment level (see Table 1). The inclusion criteria were as follows: at least 6 months after stroke, independent walking without falling regardless of walking speed, and over 70 points of Modified Barthel index. The exclusion criteria were as follows: perceptual and cognitive dysfunction and over 3 points on the Modified Ashworth Scale. Prior to the experiment, the experimenter explained all the experimental procedures to each participant and obtained informed consent.

\section{Experimental Setup and Data Collection}

Full lower body three-dimensional kinematics and EMG data were collected concurrently during overground walking from each participant. Gait kinematics were acquired using the VICON Motion Capture System (MX T-series Vicon Motion Systems Ltd, Oxford, UK), consisting of eight cameras at $100 \mathrm{~Hz}$. Surface EMG data were amplified and measured at 2000Hz with Delsys Trigno (Delsys, Inc., Natick, MA) from 16 bilateral electrodes placed on the muscles including extensor halluces longus $(E H L)$, tibialis anterior $(T A)$, soleus $(S O)$, gastrocnemius $(G A)$, vastus lateralis $(V A)$, rectus femoris $(R F)$, semitendinosus $(S M)$, and biceps femoris $(B F)$.

Each participant completed 4-6 trials of walking back and forth on a plain six meters walkway at a comfortable speed. Participants did not use any of assistive devices such as a cane, walker or ankle foot orthosis (AFO) during the recording sessions. All participants wore a 
130 harness without any body weight support for safety to catch them if needed to prevent possible

131 falls.

133 Kinematic Data Analysis

134 Visual 3D v6 Professional (C-Motion, Inc., Germantown, MD) software was used to extract 135 three-axis joint angle trajectories at pelvis and bilateral hip, knee and ankle from the marker data 136 for each trial. Custom software was written in MATLAB (Mathworks, Inc. R2016a, Natick, MA)

137 to calculate features and outcomes. The joint angle trajectories and EMG data were truncated and 138 normalized into $100 \%$ of the gait cycle from heel strike to heel strike events for each trial [17]. 139 An example of the recorded gait kinematics data of bilateral joint angle trajectories of a single 140 gait cycle in the sagittal plane is illustrated in Fig. 2.

142 Modular Neuromuscular Control Parameters

143 Area under the EMG envelope

144 The selected EMG signals from each participant were high-pass filtered at $40 \mathrm{~Hz}$ with a zero145 lag fourth-order Butterworth filter, demeaned, rectified, and low-pass filtered with a zero-lag 146 fourth-order Butterworth filter at $10 \mathrm{~Hz}$, resulting in the EMG envelope [18]. For each muscle, 147 the filtered signal was normalized to its peak value then the area under the EMG envelope curve 148 was calculated and used to represent the neuromuscular indicator as $A_{i, j}$ where $i$ can be $E H L$, $149 T A, S O, G A, V A, R F, S M$, or $B F$, and $j$ can be the unaffected $(U S)$ or affected $(A S)$ side. An 150 example area under the EMG envelope curve at $T A$ of a participant (P1) is depicted in Fig. 3. 151 Muscle Modules 
The processed EMG signals were decomposed into muscle group weightings and activation

153 timing patterns using nonnegative matrix factorization (NNMF) as previously described in [12].

154 The NNMF determined the minimum number of muscle modules based on a reconstruction 155 quality criterion: variability accounted for $(V A F) \geq 90 \%$ [12]. We additionally executed the

156 NNMF algorithm five times, considering that one to five modules were needed for the EMG

157 reconstruction. In this work, the parameters to represent the muscle modules included: number of 158 muscle modules at each side $\left(M M_{U S}\right.$ and $\left.M M_{A S}\right)$, total number of muscle modules at both sides 159 (i.e., $\left.M M_{\text {Total }}=M M_{U S}+M M_{A S}\right)$, value at $V A F \geq 90 \%\left(V A F_{U S}\right.$ and $\left.V A F_{A S}\right)$, and $V A F$ values 160 with one to five modules $\left(V A F_{U S, k}\right.$ and $V A F_{A S, k}$, where $\left.k=1, \cdots, 5\right)$.

\section{Gait Performance Measure}

\section{Gait Quality Measures}

164 We categorized the gait quality measures into spatiotemporal, limb, and joint kinematic 165 domains [3]. The parameters were extracted by imposing the average joint kinematics data of a 166 single gait cycle into a lower body model. Spatiotemporal parameters included step length $(S L)$ 167 and step time $(S T)$, defined as the linear distance between right and left feet, and the duration of 168 each step, respectively. The parameters of limb kinematics incorporate leg extension angle (LEA) 169 [19], limb length $(L L)$ [20], and footpath area $(F P A)$ [21] defined as the angle between a line 170 from hip to the foot and vertical before toe-off, the range of linear distance between hip and the 171 foot, and the area under the foot pattern from hip sagittal plain during gait cycle, respectively.

172 The parameters of joint kinematics were defined as the range of motion (RoM) of selected joints 173 including all rotations of hip, knee flex/extension, and ankle dorsi/plantarflexion.

174 The symmetry index metric [22] was used to evaluate the gait quality given by 


$$
S I_{n}=\frac{U S_{n}-A S_{n}}{0.5\left(U S_{n}+A S_{n}\right)}
$$

176 where $U S_{n}$ and $A S_{n}$ are the $n^{\text {th }}$ gait parameter of the unaffected and affected sides, respectively, 177 and $n$ can be the aforementioned spatiotemporal, limb and joint kinematic parameters. The value 178 is always between -2 to 2 , and a positive (or negative) value indicates $U S>A S$ (or vice versa) 179 [22]. Note that the symmetry index, $S I_{n}=0$, when perfect symmetry.

\section{Functional Gait Measure}

181

182

183

184
For the functional gait measure, we selected gait speed $(G S)$ because speed is a wellaccepted indicator of gait performance after stroke [23].

\section{Statistical Analysis}

Subjects were classified into two groups based on the criterion of total number of muscle modules at both sides equal or less than four (i.e., $M M_{\text {Total }} \leq 4$ ), which was determined by rounding the average total number of muscle modules $\left(M M_{\text {Average }}=4.37\right)$ of all subjects. A two-sided, two-sample t-test was used to test the difference of gait performance measures between the two groups with a significance level of $\alpha<0.05$.

Second, a stepwise multiple regression analysis was used to investigate which combination of the neuromuscular parameters was best associated with gait quality measures with a significance level of $\alpha<0.05$. A total of 32 aforementioned independent variables including the modular neuromuscular control parameters and a functional gait measure (i.e., speed) were selected. The dependent variables were the gait quality measures including symmetry index of spatiotemporal, limb and joint kinematic parameters. A preliminary analysis was conducted using a linear regression on each considered independent variable and each dependent variable to minimize the number of independent variables and to simplify the final model as possible. Only 
198 those independent variable candidates with $p$-value $\leq 0.05$ were selected as input for the

199 stepwise multiple regression analysis.

200

201 Results

202 Classification based on Number of Muscle Modules

203 The average total number of modules $\left(M M_{\text {Total }}\right)$ was 4.37 with a minimum and maximum of 204 two and six modules, respectively. We classified subjects whose total number of muscle modules 205 were equal or less than four (i.e., $\left.M M_{\text {Total }} \leq 4\right)$ into Group 1 and the rest of the subjects (i.e., $206 M M_{\text {Total }}>4$ ) into Group 2. A total seven of subjects were classified into Group 1 (subject ID: 3, $2074,5,6,8,10$, and 13), and nine subjects were included into Group 2 (subject ID: 1, 2, 7, 9, 11, 208 12, 14, 15, and 16). All results are summarized in Table 2.

209 A two-sided, two-sample t-test was used to evaluate the significant difference of each gait 210 parameter (i.e., symmetry index) between the two groups. Among all the gait parameters, 211 symmetry indices of $L L(p<0.01)$ and FPA $(p<0.01)$ from limb kinematics and hip flex/extension $212(p<0.05)$, hip abd/adduction $(p<0.01)$, and knee flex/extension $(p<0.01)$ from joint kinematics 213 revealed a significant difference between the two groups. While gait speed $(p=0.07)$ and ankle 214 dorsi/plantarflexion $(p=0.06)$ revealed a considerably strong difference, these parameters 215 including other parameters did not reveal a significant difference between the two groups (see 216 Table 3).

217 We additionally fitted a linear regression model on the symmetry index of those significant 218 parameters including $L L(p<0.05), F P A(p<0.05)$, hip flex/extension $(p=0.10)$, hip abd/adduction $219(p<0.05)$, and knee flex/extension $(p<0.05)$ across the total number of modules. All parameters 
except for hip flex/extension still revealed a significant trend in the slope of the linear regression models (See Fig. 4).

\section{Stepwise Regression to Predict Gait Features}

\section{Prediction of Spatiotemporal Parameters}

For $S L$, the stepwise regression analysis selected $V A F_{A S}\left(\beta_{1}=-10.29\right)$ and $A_{R F, A S}\left(\beta_{2}=\right.$ $14.01)$ as independent variables. The model revealed a statistically significant relationship $(F=$ 8.26, $p<0.001)$ and accounted for approximately $56 \%$ of the variance of $S L\left(R^{2}=0.56\right.$, Adjusted $\left.R^{2}=0.49\right)$. In contrast, none of the independent variable candidates had a $p$-value $<$ 0.05 for $S T$ (see Table 4). The visualization of the regression model with selected independent variables is shown in Fig. 5 (top left).

\section{Prediction of Limb Kinematic Parameters}

For $L E A$, the stepwise regression analysis selected $A_{R F, A S}\left(\beta_{1}=15.18\right)$ as an independent variable, was statistically significant $(F=9.16, p<0.001)$, and accounted for approximately $40 \%$ of the variance $\left(R^{2}=0.40\right.$, Adjusted $\left.R^{2}=0.35\right)$. For $L L$ and FPA, both stepwise regression analyses selected $V A F_{A S, 3}\left(\beta_{1}=17.82\right.$ for $L L$ and $\beta_{1}=20.52$ for $\left.F P A\right)$ as an independent variable. The fitted models were statistically significant $(F=5.96, p<0.05$ for $L L$ and $F=7.73, p<0.05$ for $F P A$ ) and accounted for approximately $30 \%$ and $36 \%$ of the variance of $L L$ and FPA, respectively $\left(R^{2}=0.30\right.$, Adjusted $R^{2}=0.25$ for $L L$ and $R^{2}=0.36$, Adjusted $R^{2}=0.31$ for $F P A$ ). The results are summarized in Table 4 with the visualization of the regression models with selected independent variables shown in Fig. 5 (top right three).

\section{Prediction of Joint Kinematic Parameters}


For the hip flex/extension, the stepwise regression analysis selected $V A F_{U S, 1}\left(\beta_{1}=2.27\right)$

243

244

245

246

247

248

249

250

251

252

253

254

255

256

257

258

259

260

261

262

263

264

and $A_{R F, U S}\left(\beta_{2}=4.91\right)$ as the independent variables and were statistically significant $(F=13.0$, $p<0.001)$ accounting for approximately $67 \%$ of the variance $\left(R^{2}=0.67\right.$, Adjusted $R^{2}=$ 0.62). For the hip abd/adduction, the stepwise regression analysis selected $V A F_{U S, 1}\left(\beta_{1}=2.56\right)$ and $A_{V L, A S}\left(\beta_{2}=1.76\right)$ as the independent variables and were statistically significant $(F=8.39$, $p<0.01)$ accounting for approximately $56 \%$ of the variance $\left(R^{2}=0.56\right.$, Adjusted $\left.R^{2}=0.50\right)$. For the hip int/external rotation, the stepwise regression analysis selected $A_{T A, U S}\left(\beta_{1}=-2.25\right)$ as the independent variable and was statistically significant $(F=8.39, p<0.01)$ accounting for approximately $39 \%$ of the variance $\left(R^{2}=0.39\right.$, Adjusted $\left.R^{2}=0.35\right)$. For the knee flex/extension, the stepwise regression analysis selected $\operatorname{VAF}_{A S, 3}\left(\beta_{1}=19.24\right)$ as the independent variable. The fitted model was statistically significant $(F=9.91, p<0.01)$ and accounted for approximately $41 \%$ of the variance of the knee flex/extension $\left(R^{2}=0.41\right.$, Adjusted $\left.R^{2}=0.37\right)$. Finally, for the ankle dorsi/plantarflexion, none of the independent variable candidates had a $p$-value $<0.05$. (See Table 4 ). The visualization of the regression models with selected independent variables is shown in Fig. 5 (bottom row).

\section{Discussion}

The major goal of this study was to delineate the causal relations of modular neuromuscular control parameters and quality of movement during gait after stroke. The main findings were as follows: first, post-stroke individuals with a reduced number of muscle modules exhibit greater asymmetry in gait quality measures, particularly in kinematics level. Second, the gait quality measures were predictable with modular neuromuscular control parameters extracted from the EMG data. Specifically, those parameters were variability accounted for $(V A F)$ information from 
265 muscle modules (i.e., $V A F_{A S}, V A F_{A S, 3}$, and $V A F_{U S, 1}$ ) and area under the EMG envelope curves

266 from rectus femoris, vastus lateralis, and tibialis anterior muscles (i.e., $A_{R F, A S}, A_{R F, U S}, A_{V L, A S}$,

267 and $\left.A_{T A, U S}\right)$. To our knowledge, this work is a novel in the fact that it integrates detailed gait

268 quality measures with the neuromuscular control framework. The results in this study offer

269 preliminary evidence justifying that the modular neuromuscular framework can be a useful

270 predictor of gait quality measures and help to understand the underlying causality of disturbances

271 in gait quality after stroke.

272 The analysis of the muscle modules has become a more popular tool to describe the

273 neuromotor control of multi-limb movement such as gait after stroke [12]. For the analysis, many

274 previous studies classified muscle modules into paretic and non-paretic sides [12], [24], [25].

275 While this is a reasonable separation given that most stroke populations exhibit a hemiplegic

276 gait, our rationale was that walking is essentially the performance of an inter-coordinated

277 behavior between both legs. Thus, we analyzed the data from a different perspective of

278 classifying participant groups based on the total number of muscle modules of both sides. Our

279 data revealed that there is a significant difference in gait quality measures between the groups,

280 indicating a link between the total number of modules and the gait quality. This result is

281 consistent with a previous study that found merged muscle module is critical to poor walking

282 performance [25]. This also justifies the investigation of therapeutic interventions that can

283 increase the number of modules to improve the gait quality after stroke [14].

284 One thing to note is that most participants in our data had a relatively severe gait impairment

285 with slow walking speed (average speed: $0.29 \pm 0.13 \mathrm{~m} / \mathrm{s}$, see Table 1) [26]. Nevertheless, we

286 found significant differences in gait quality measures between the groups and those differences

287 were mostly observed in the kinematic parameters (see Table 3). However, none of the 
288 parameters from spatiotemporal characteristics and functional gait measure (i.e., speed) revealed 289 a significant difference between the groups. These results correspond with previous research that 290 found post-stroke individuals exhibit significant asymmetry in joint kinematics with greater 291 inter-individual variability than spatiotemporal characteristics [9]. Given that significance was 292 observed between the groups within a severe population in our data, we speculate to find a more 293 distinct trend with a larger sample size with various severity levels including mild to moderately 294 impaired stroke population.

295 A recent study attempted to combine parameters from muscle modules and gait analysis 296 (i.e., spatiotemporal and joint kinematics) to predict functional outcomes such as walking speed 297 [24]. While this study successfully predicted locomotor function with combined biomechanical 298 and neuromuscular measures, the relations between those measures, which would more likely to 299 have direct causal relations, were not analyzed. Thus, we used the stepwise multiple regression 300 approach to find the optimal linear regression model that predicts the gait quality measures with 301 the input modular neuromuscular control parameters. We found that all symmetry indices of gait 302 quality measures, except for step time and ankle dorsi/plantarflexion, were predictable with the 303 neuromuscular modular control parameters with significant associations (see Table 4). Common 304 predictors included quadriceps muscles (i.e., rectus femoris and vastus lateralis) and information 305 from variability accounted for $(V A F)$ in the regression models. The inclusion of quadriceps were 306 expected as these muscles have long been believed to be key contributors to hip and knee motion 307 during gait [27]. $V A F$ s were also commonly selected because these parameters are critical 308 information determining the number of muscle modules [24]. On the other hand, it is unclear 309 why the tibialis anterior muscle appeared to be negatively associated with hip internal/external 310 rotation given that this muscle is known as ankle dorsiflexor. This result may be related to 
311 abnormal compensatory coupling between irrelevant muscles due to neurological impairment

312 [28]. However, further research is needed where the EMG measures at other locations such as

313 hip lateral rotator group muscles are added to investigate this connection, which may indeed be

314 epiphenomenal. Overall, these results suggest causal relations exist between the neuromuscular

315 control framework and the gait quality measures.

316 One possible therapeutic application of this causal relationship would be using the concept

317 of neuromuscular and gait quality parameters as outcome measures to assess gait impairment

318 after stroke. An example of previous research would be a locomotor intervention study that

319 applied muscle modules as pre- and post-therapy outcome measures [14], [29]. These studies

320 found increase in number of muscle modules and improvements in quality of modular

321 organization (i.e., timing and compositions) as well as clinical measures such as gait speed after

322 a locomotor rehabilitation therapy. This indicates that therapeutic interventions can change or

323 improve the neuromuscular control framework, but the influence of these interventions on

324 detailed movement quality was not reported in these studies. Thus, we expect the application of

325 the neuromuscular and gait quality parameters as outcome measures will fill the gap and provide

326 a more comprehensive characterization of gait recovery post-stroke.

327 The purpose of this proof-of-concept study was to provide initial evidence of causal

328 relations between gait quality and neuromuscular parameters for chronic post-stroke individuals.

329 However, this study was limited to a small sample size of 16 patients with a restricted range of

330 impairment level; only severely impaired individuals with a slow walking speed participated in

331 this work. However, our data still showed clear initial results on the relative importance of

332 several predictors, providing the significant causal relationships exist between neuromuscular

333 control framework and gait quality measures. We expect this methodology can be potentially 
334 used for gait training research or in clinical practice to understand impairments related to gait 335 function.

\section{Conclusions}

The purpose of this study was to investigate the influence of modular neuromuscular control

339 framework on gait quality measures. We observed that chronic post-stroke individuals with a 340 reduced number of muscle modules exhibit a greater deficit in gait quality measures, particularly

341 in kinematics level. We also found that the gait quality measures are predictive with the input

342 modular neuromuscular control variables. We conclude that there exists causal relations between

343 the neuromuscular control framework and gait quality measures. These promising results justify

344 further research with a larger post-stroke population and expanded range of impairment level for 345 more reliable generalization.

\section{List of abbreviations}

348 AFO: ankle foot orthosis; $A S$ : affected side; $B F$ : biceps femoris; $E H L$ : extensor halluces longus; 349 EMG: electromyography; FPA: footpath area; GA: gastrocnemius; GS: gait speed; $L E A$ : leg 350 extension angle; $L L$ : limb length; $M M$ : muscle module; NNMF: nonnegative matrix 351 factorization; $R F$ : rectus femoris; RoM: range of motion; $S I$ : symmetry index; SL: step length;

$352 S M$ : semitendinosus; $S O$ : soleus; $S T$ : step time; $T A$ : tibialis anterior; $U S$ : unaffected side; $V A$ :

353 vastus lateralis; $V A F$ : variability accounted for 
356 This study was approved by Institutional Review Board of the Korea Advanced Institute of

357 Science and Technology (KH2019-05). All subjects gave written informed consent prior to data 358 collection.

359

360 Consent for publication

361 Participants gave written informed consent to data treatment in this research study and

362 permission to publish anonymous data and results.

363

364 Availability of data and materials

365 The data collected in this study are available from the corresponding author on reasonable

366 request.

367

368 Competing interests

369 The authors declare that they have no competing interests.

370

371 Funding

372 This paper is based on research that was conducted as part of the KAIST funded Global

373 Singularity Research Program for 2019.

374

375 Author's contribution

376 SYS drafted the complete manuscript, performed data post-processing and statistical data

377 analysis. YK participated in conducting experimental data collection, recruited subjects and 
378 managed IRB approvals. AJ and HSP conceived the whole idea, managed IRB approvals and 379 helped in finalizing the manuscript. All authors read and approved the final manuscript.

380

\section{Acknowledgements}

382 None. 


\section{References}

[1] J. K. Tilson et al., "Meaningful gait speed improvement during the first 60 days poststroke: minimal clinically important difference," Physical therapy, vol. 90, no. 2, pp. 196-208, 2010.

[2] P. L. Enright, “The six-minute walk test," Respiratory care, vol. 48, no. 8, pp. 783-785, 2003.

[3] S. Y. Shin, R. K. Lee, P. Spicer, and J. Sulzer, "Does kinematic gait quality improve with functional gait recovery? A longitudinal pilot study on early post-stroke individuals," Journal of Biomechanics, p. 109761, 2020.

[4] V. Weerdesteyn, M. de Niet, H. J. van Duijnhoven, and A. C. Geurts, "Falls in individuals with stroke," Journal of Rehabilitation Research \& Development, vol. 45, no. 8, pp. 11951214, 2008.

[5] J. M. Finley, A. J. Bastian, and J. S. Gottschall, "Learning to be economical: the energy cost of walking tracks motor adaptation," The Journal of physiology, vol. 591, no. 4, pp. 1081-1095, 2013.

[6] M. F. Levin, J. A. Kleim, and S. L. Wolf, "What do motor 'recovery' and 'compensation' mean in patients following stroke?," Neurorehabilitation and neural repair, vol. 23, no. 4, pp. 313-319, 2009.

[7] T. A. Jones, "Motor compensation and its effects on neural reorganization after stroke," Nature Reviews Neuroscience, vol. 18, no. 5, p. 267, 2017.

[8] K. K. Patterson, W. H. Gage, D. Brooks, S. E. Black, and W. E. McIlroy, "Evaluation of gait symmetry after stroke: a comparison of current methods and recommendations for standardization," Gait \& posture, vol. 31, no. 2, pp. 241-246, 2010.

[9] S. M. Woolley, "Characteristics of gait in hemiplegia," Topics in stroke rehabilitation, vol. 7, no. 4, pp. 1-18, 2001.

[10] J. Perry and J. M. Burnfield, "Gait analysis: normal and pathological function," 1992, Accessed: Oct. 09, 2016. [Online]. Available: http://www.healio.com/books/healthprofessions/physical-therapy/\%7Bec12a908-c0f3-42af-8b11-c3fc73727078\%7D/gaitanalysis-normal-and-pathological-function-second-edition.

[11] G. Cappellini, Y. P. Ivanenko, R. E. Poppele, and F. Lacquaniti, "Motor patterns in human walking and running," Journal of neurophysiology, vol. 95, no. 6, pp. 3426-3437, 2006.

[12] D. J. Clark, L. H. Ting, F. E. Zajac, R. R. Neptune, and S. A. Kautz, "Merging of healthy motor modules predicts reduced locomotor performance and muscle coordination complexity post-stroke," Journal of neurophysiology, vol. 103, no. 2, pp. 844-857, 2010.

[13] L. H. Ting et al., "Neuromechanical principles underlying movement modularity and their implications for rehabilitation," Neuron, vol. 86, no. 1, pp. 38-54, 2015.

[14] R. L. Routson, D. J. Clark, M. G. Bowden, S. A. Kautz, and R. R. Neptune, "The influence of locomotor rehabilitation on module quality and post-stroke hemiparetic walking performance," Gait \& posture, vol. 38, no. 3, pp. 511-517, 2013.

[15] S. Li, G. E. Francisco, and P. Zhou, "Post-stroke hemiplegic gait: new perspective and insights," Frontiers in physiology, vol. 9, p. 1021, 2018.

[16] J. M. Winters, S. L. Woo, and I. Delp, Multiple muscle systems: Biomechanics and movement organization. Springer Science \& Business Media, 2012. 
[17] J. A. Zeni Jr, J. G. Richards, and J. S. Higginson, "Two simple methods for determining gait events during treadmill and overground walking using kinematic data," Gait \& posture, vol. 27, no. 4, pp. 710-714, 2008.

[18] P. Konrad, "The abc of emg," A practical introduction to kinesiological electromyography, vol. 1 , no. 2005, pp. 30-5, 2005.

[19] C. L. Peterson, J. Cheng, S. A. Kautz, and R. R. Neptune, "Leg extension is an important predictor of paretic leg propulsion in hemiparetic walking," Gait \& posture, vol. 32, no. 4, pp. 451-456, 2010.

[20] Y.-H. Chang, A. G. Auyang, J. P. Scholz, and T. R. Nichols, "Whole limb kinematics are preferentially conserved over individual joint kinematics after peripheral nerve injury," Journal of Experimental Biology, vol. 212, no. 21, pp. 3511-3521, 2009.

[21] S. K. Banala, S. H. Kim, S. K. Agrawal, and J. P. Scholz, "Robot assisted gait training with active leg exoskeleton (ALEX)," IEEE Transactions on Neural Systems and Rehabilitation Engineering, vol. 17, no. 1, pp. 2-8, 2009.

[22] K. K. Patterson, W. H. Gage, D. Brooks, S. E. Black, and W. E. McIlroy, "Evaluation of gait symmetry after stroke: a comparison of current methods and recommendations for standardization," Gait \& posture, vol. 31, no. 2, pp. 241-246, 2010.

[23] M. G. Bowden, C. K. Balasubramanian, A. L. Behrman, and S. A. Kautz, "Validation of a speed-based classification system using quantitative measures of walking performance poststroke," Neurorehabilitation and neural repair, vol. 22, no. 6, pp. 672-675, 2008.

[24] F. O. Barroso et al., "Combining muscle synergies and biomechanical analysis to assess gait in stroke patients," Journal of Biomechanics, vol. 63, pp. 98-103, 2017.

[25] L. G. Brough, S. A. Kautz, M. G. Bowden, C. M. Gregory, and R. R. Neptune, "Merged plantarflexor muscle activity is predictive of poor walking performance in post-stroke hemiparetic subjects," Journal of biomechanics, vol. 82, pp. 361-367, 2019.

[26] A. Schmid et al., "Improvements in speed-based gait classifications are meaningful," Stroke, vol. 38, no. 7, pp. 2096-2100, 2007.

[27] S. Sipilä and H. Suominen, "Knee extension strength and walking speed in relation to quadriceps muscle composition and training in elderly women," Clinical Physiology, vol. 14, no. 4, pp. 433-442, 1994.

[28] T. Akbas, R. Neptune, and J. Sulzer, "Neuromusculoskeletal simulation reveals abnormal rectus femoris-gluteus medius coupling in post-stroke gait," Frontiers in neurology, vol. 10, p. 301, 2019.

[29] M. M. Ardestani, C. R. Kinnaird, C. E. Henderson, and T. G. Hornby, "Compensation or recovery? Altered kinetics and neuromuscular synergies following high-intensity stepping training poststroke," Neurorehabilitation and neural repair, vol. 33, no. 1, pp. 47-58, 2019. 


\section{Figure Legends}

465 Figure 1. Overview of the study.

466

467 Figure 2. Example joint angle trajectories of a single gait cycle (heel strike to heel strike) in

468 sagittal plane, hip flexion/extension (left), knee flexion/extension (middle), and ankle

469 dorsi/plantarflexion (right).

470

471 Figure 3. Example area under the EMG envelope curve of tibialis anterior muscle from

472 participant P1. A.U. in the y-axis indicates arbitrary unit.

473

474 Figure 4. Symmetry index $(S I)$ of significant gait parameters including limb length $(L L)$,

475 footpath area $(F P A)$, hip flex/extension (Hip FE), hip abd/adduction (Hip $A A)$ and knee

476 flex/extension (Knee $F E$ ) with increased total number of muscle modules at both sides

$477\left(M M_{\text {Total }}\right)$. Linear regression models (bottom, right) were fitted on each gait parameter. The

478 shaded area indicates $95 \%$ confidence interval.

479

480 Figure 5. Regression models with selected independent variables (see Table 3) from stepwise

481 multiple regression. Note that a plane in three-dimensional space is illustrated with two

482 independent variables for step length, hip flex/extension and hip abd/adduction. 
484 Table 1. Demographics and clinical characteristics of the participants.

\begin{tabular}{|c|c|c|c|c|c|c|c|c|c|c|}
\hline ID & $\begin{array}{c}\text { Age } \\
\text { (years) }\end{array}$ & Sex & $\begin{array}{l}\text { Height } \\
(\mathrm{cm})\end{array}$ & $\begin{array}{c}\text { Weight } \\
(\mathrm{kg})\end{array}$ & $\begin{array}{c}\text { Side } \\
\text { affected }\end{array}$ & $\begin{array}{c}\text { Time after } \\
\text { stroke (years) }\end{array}$ & $\begin{array}{c}\text { Type of } \\
\text { lesion }\end{array}$ & FMA & FIM & $\begin{array}{c}\text { Speed } \\
(\mathrm{m} / \mathrm{s})\end{array}$ \\
\hline $\mathrm{P} 1$ & 59 & $\mathrm{M}$ & 170 & 69 & $\mathrm{R}$ & 4 & Ischemic & 21 & 11 & 0.29 \\
\hline $\mathrm{P} 2$ & 61 & $\mathrm{M}$ & 169 & 72 & $\mathrm{~L}$ & 3 & Ischemic & 16 & 9 & 0.27 \\
\hline P3 & 70 & $\mathrm{M}$ & 158 & 69 & $\mathrm{R}$ & 2 & Ischemic & 12 & 12 & 0.16 \\
\hline $\mathrm{P} 4$ & 55 & $\mathrm{~F}$ & 158 & 60 & $\mathrm{~L}$ & 11 & Hemorrhage & 22 & 14 & 0.24 \\
\hline $\mathrm{P} 5$ & 59 & $\mathrm{M}$ & 176.5 & 66 & $\mathrm{R}$ & 3 & Ischemic & 13 & 8 & 0.21 \\
\hline P6 & 73 & $\mathrm{M}$ & 170 & 74 & Both & 21 & Hemorrhage & 22 & 3 & 0.18 \\
\hline P7 & 75 & $\mathrm{~F}$ & 153 & 49 & $\mathrm{R}$ & 5 & Ischemic & 25 & 12 & 0.20 \\
\hline P8 & 75 & $\mathrm{M}$ & 164 & 62 & $\mathrm{R}$ & 15 & Ischemic & 18 & 13 & 0.31 \\
\hline P9 & 68 & $\mathrm{M}$ & 172 & 83 & $\mathrm{~L}$ & 10 & Ischemic & 26 & 13 & 0.46 \\
\hline $\mathrm{P} 10$ & 36 & $\mathrm{M}$ & 173 & 80 & $\mathrm{~L}$ & 1 & Ischemic & 17 & 7 & 0.15 \\
\hline $\mathrm{P} 11$ & 77 & $\mathrm{M}$ & 169 & 58 & $\mathrm{R}$ & 25 & Ischemic & 10 & 12 & 0.26 \\
\hline $\mathrm{P} 12$ & 55 & $\mathrm{M}$ & 177 & 84 & $\mathrm{~L}$ & 13 & Ischemic & 15 & 14 & 0.37 \\
\hline $\mathrm{P} 13$ & 47 & $\mathrm{~F}$ & 163 & 57 & $\mathrm{R}$ & 4 & Hemorrhage & 19 & 13 & 0.29 \\
\hline $\mathrm{P} 14$ & 64 & $\mathrm{M}$ & 172 & 81 & $\mathrm{R}$ & 27 & Ischemic & 31 & 14 & 0.49 \\
\hline $\mathrm{P} 15$ & 62 & $\mathrm{~F}$ & 156 & 57 & $\mathrm{~L}$ & 15 & Hemorrhage & 16 & 11 & 0.13 \\
\hline $\mathrm{P} 16$ & 70 & $\mathrm{M}$ & 171 & 83 & $\mathrm{R}$ & 18 & Ischemic & 27 & 12 & 0.58 \\
\hline
\end{tabular}


488 Table 2. Classification of the subject groups based on the total number of muscle modules at 489 both sides

\begin{tabular}{|c|c|c|c|l|c|}
\hline Group & $M M_{\text {Total }}$ & $M M_{U S}$ & $M M_{A S}$ & \multicolumn{1}{|c|}{ Subject ID } & $\begin{array}{c}\text { Number of } \\
\text { Subjects }\end{array}$ \\
\hline \multirow{3}{*}{ Group 1 } & 2 & 1 & 1 & P6 & 1 \\
\cline { 2 - 6 } & 3 & 2 & 1 & P4, P10 & 2 \\
\cline { 2 - 6 } & 4 & 2 & 2 & P3, P5, P8, P13 & 4 \\
\hline \multirow{3}{*}{ Group 2 } & 5 & 2 & 3 & P7 & 7 \\
\cline { 2 - 6 } & 5 & 3 & 2 & P1, P2, P9, P11, P12, P14, P15 & 1 \\
\hline
\end{tabular}

490

$M M$ - number of muscle modules, $U S$ - unaffected side, $A S$ - affected side

491 
492 Table 3. Results of the two-sided, two-sample t-tests of gait performance measures between two 493 groups

\begin{tabular}{|c|c|c|c|c|r|}
\hline Gait Features & SI Parameters [\%] & Group1 & Group2 & 95\% CI & p-value \\
\hline Functional & Speed & $0.22 \pm 0.06$ & $0.34 \pm 0.15$ & {$[-0.25,0.01]$} & 0.07 \\
\hline \multirow{2}{*}{$\begin{array}{c}\text { Spatiotemporal } \\
\text { characteristics }\end{array}$} & Step length & $-0.34 \pm 0.34$ & $-0.44 \pm 0.32$ & {$[-0.27,0.45]$} & 0.59 \\
\hline \multirow{3}{*}{$\begin{array}{c}\text { Limb } \\
\text { kinematics }\end{array}$} & Step duration & $-0.19 \pm 0.15$ & $-0.15 \pm 0.08$ & {$[-0.16,0.09]$} & 0.58 \\
\cline { 2 - 6 } & Leg extension angle & $-0.05 \pm 0.27$ & $-0.16 \pm 0.35$ & {$[-0.23,0.45]$} & 0.50 \\
\cline { 2 - 6 } & Limb length & $0.84 \pm 0.10$ & $0.38 \pm 0.35$ & {$[0.17,0.76]$} & $<0.01$ \\
\cline { 2 - 6 } & Footpath area & $1.04 \pm 0.13$ & $0.51 \pm 0.36$ & {$[0.22,0.84]$} & $<0.01$ \\
\cline { 2 - 6 } Joint \\
\cline { 2 - 6 } kinematics & Hip flex/ex & $0.67 \pm 0.27$ & $0.38 \pm 0.18$ & {$[0.04,0.53]$} & $<0.05$ \\
\cline { 2 - 6 } & Hip abd/add & $0.31 \pm 0.25$ & $-0.02 \pm 0.16$ & {$[0.11,0.55]$} & $<0.01$ \\
\cline { 2 - 6 } & Hip int/ext ro. & $-0.05 \pm 0.14$ & $-0.02 \pm 0.23$ & {$[-0.25,0.18]$} & 0.75 \\
\cline { 2 - 6 } & Knee flex/ex & $0.56 \pm 0.23$ & $0.15 \pm 0.23$ & {$[0.15,0.65]$} & $<0.01$ \\
\cline { 2 - 6 } & Ankle dorsi/plantar & $0.37 \pm 0.36$ & $0.05 \pm 0.25$ & {$[-0.01,0.64]$} & 0.06 \\
\hline
\end{tabular}


Table 4. Results of the stepwise multiple regression models with the dependent variable of symmetry ratio of gait quality measures and independent variable of modular neuromuscular parameters

\begin{tabular}{|c|c|c|c|c|c|c|}
\hline Gait Features & SI parameters [\%] & Multiple regression model & F-value & p-value & $R^{2}$ & $\begin{array}{l}\text { adjusted } \\
R^{2}\end{array}$ \\
\hline \multirow{2}{*}{$\begin{array}{l}\text { Spatiotemporal } \\
\text { characteristics }\end{array}$} & Step length & $\begin{aligned} S I_{S L}=8.422- & 10.29 V A F_{A S} \\
& +14.01 A_{R F, A S}\end{aligned}$ & 8.26 & $<0.001$ & 0.56 & 0.49 \\
\hline & Step time & - & - & - & - & - \\
\hline \multirow{3}{*}{$\begin{array}{l}\text { Limb } \\
\text { kinematics }\end{array}$} & Leg extension angle & $S I_{L E A}=-0.98+15.18 A_{R F, A S}$ & 9.16 & $<0.001$ & 0.40 & 0.35 \\
\hline & Limb length & $S I_{L L}=-16.52+17.82 V A F_{A S, 3}$ & 5.96 & $<0.05$ & 0.30 & 0.25 \\
\hline & Footpath area & $S I_{F P A}=-18.98+20.52 V A F_{A S, 3}$ & 7.73 & $<0.05$ & 0.36 & 0.31 \\
\hline \multirow{5}{*}{$\begin{array}{c}\text { Joint } \\
\text { kinematics }\end{array}$} & Hip flex/ex & $\begin{aligned} S I_{H i p, F E}=-1.82 & +2.27 V A F_{U S, 1} \\
& +4.91 A_{R F, U S}\end{aligned}$ & 13.0 & $<0.001$ & 0.67 & 0.62 \\
\hline & Hip abd/add & $\begin{aligned} S I_{H i p, A A}=-2.19+ & 2.56 V A F_{U S, 1} \\
& +1.76 A_{V L, A S}\end{aligned}$ & 8.39 & $<0.01$ & 0.56 & 0.50 \\
\hline & Hip int/ext ro. & $S I_{H i p, I E}=0.46-2.25 A_{T A, U S}$ & 8.39 & $<0.01$ & 0.39 & 0.35 \\
\hline & Knee flex/ex & $S I_{K n e e, F E}=-18.1+19.24 V A F_{A S, 3}$ & 9.91 & $<0.01$ & 0.41 & 0.37 \\
\hline & Ankle dorsi/plantar & - & - & - & - & - \\
\hline
\end{tabular}


Fig 1.
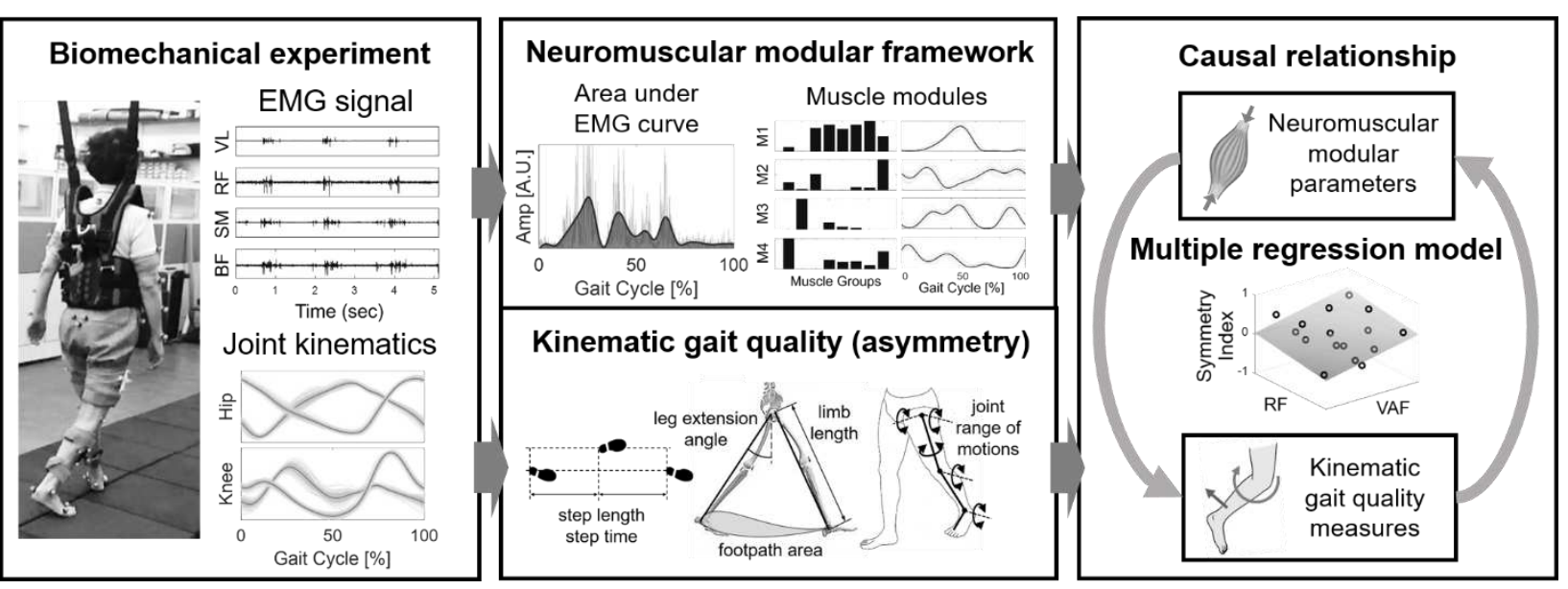
Fig 2.
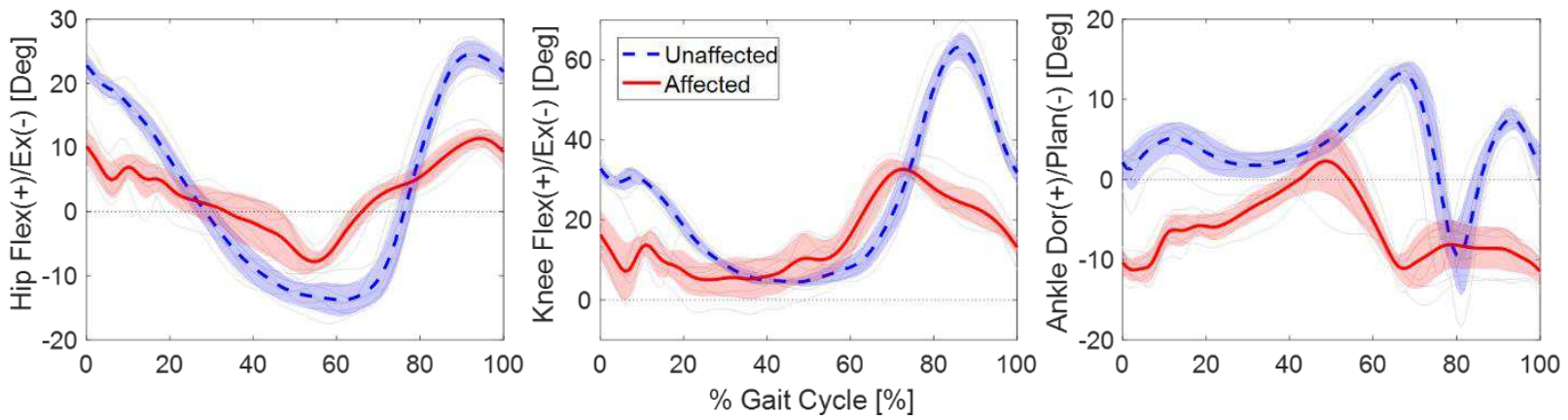

508

509 
Fig 3.

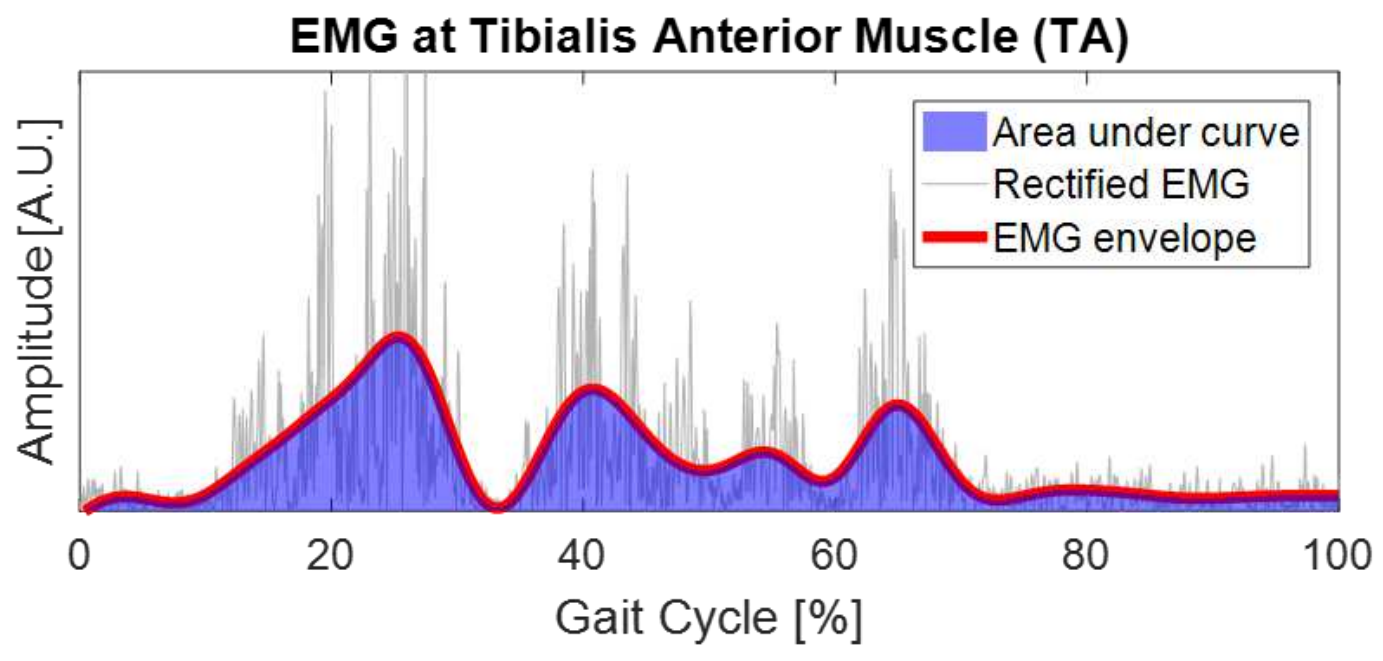

511

512 
$513 \quad$ Fig 4.
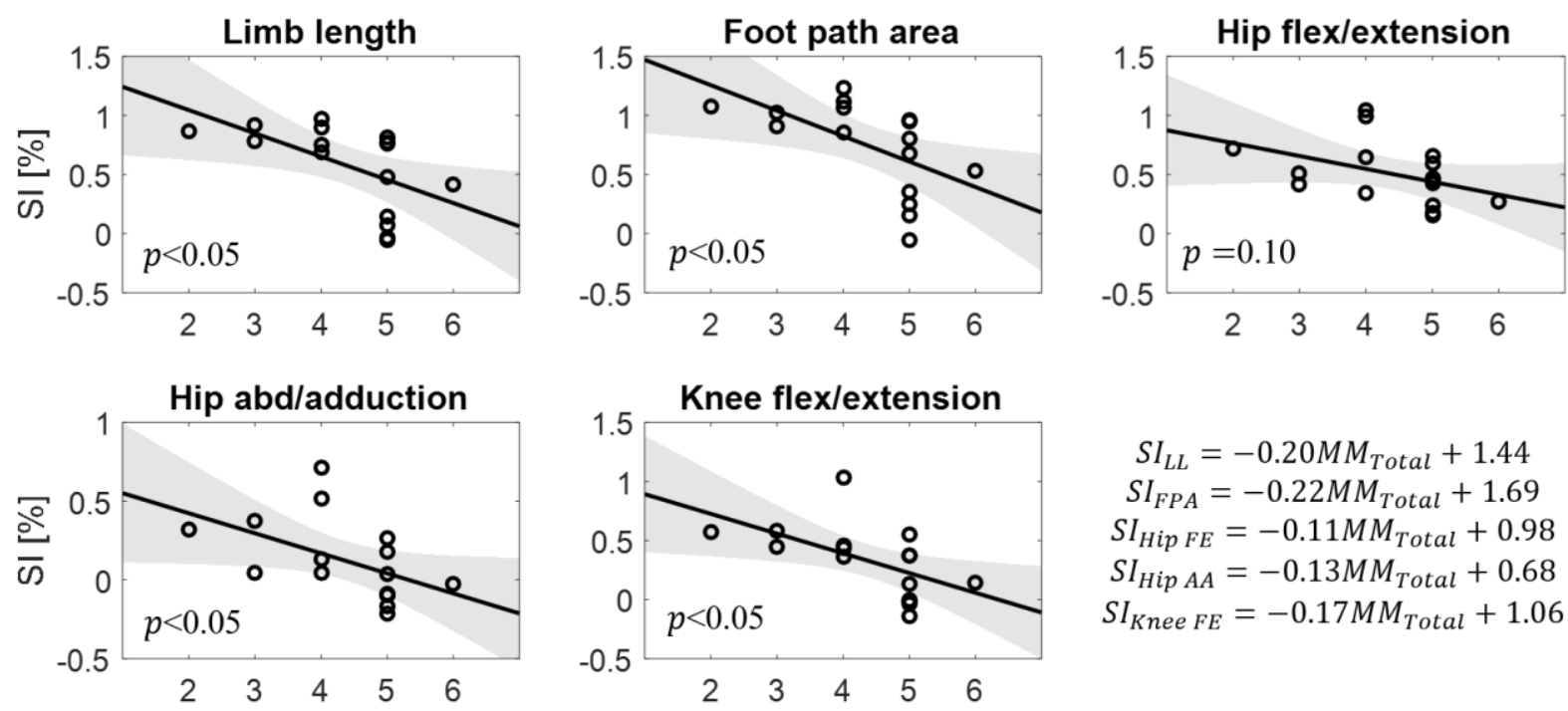

514

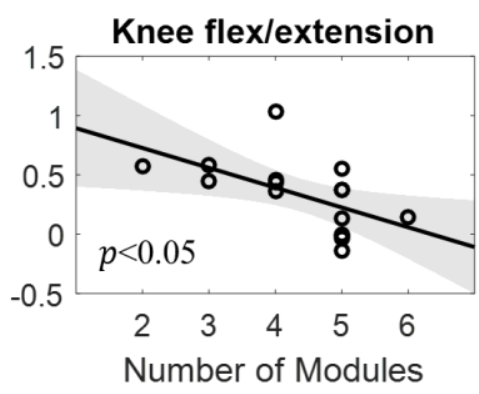

$S I_{L L}=-0.20 M M_{\text {Total }}+1.44$
$S I_{F P A}=-0.22 M M_{\text {Total }}+1.69$
$S I_{\text {Hip FE }}=-0.11 M M_{\text {Total }}+0.98$
$S I_{\text {Hip AA }}=-0.13 M M_{\text {Total }}+0.68$
$S I_{\text {Knee FE }}=-0.17 M M_{\text {Total }}+1.06$

515 
$517 \quad$ Fig. 5.
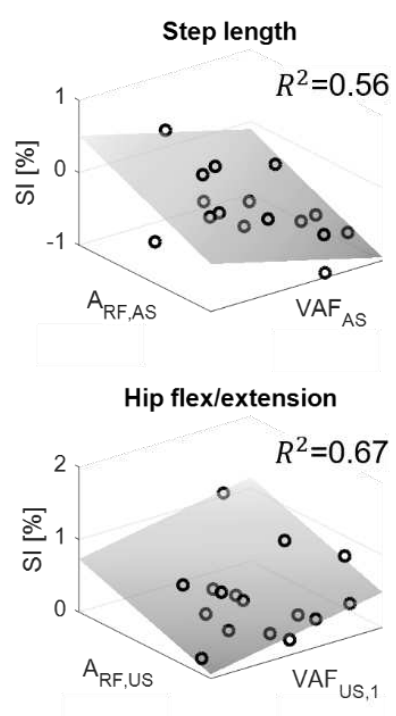

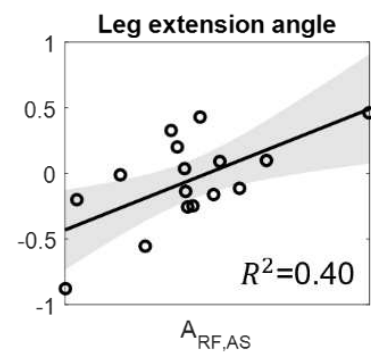

Hip abd/adduction

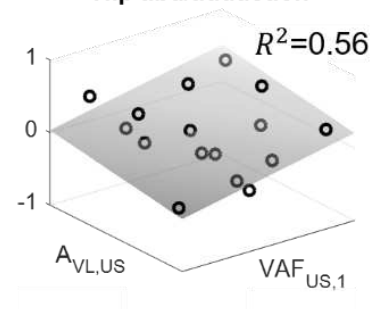

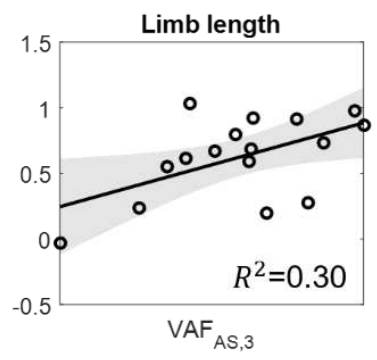
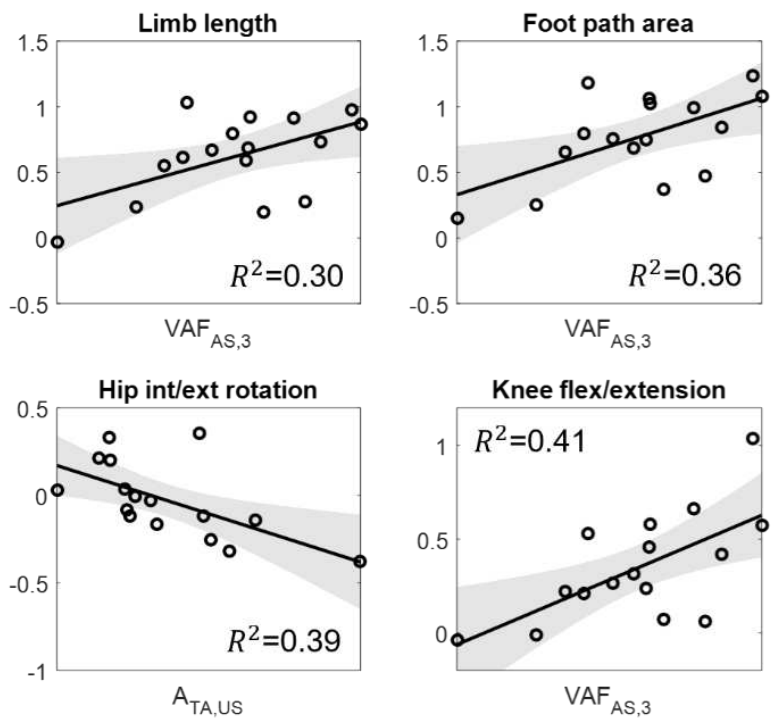
Figures
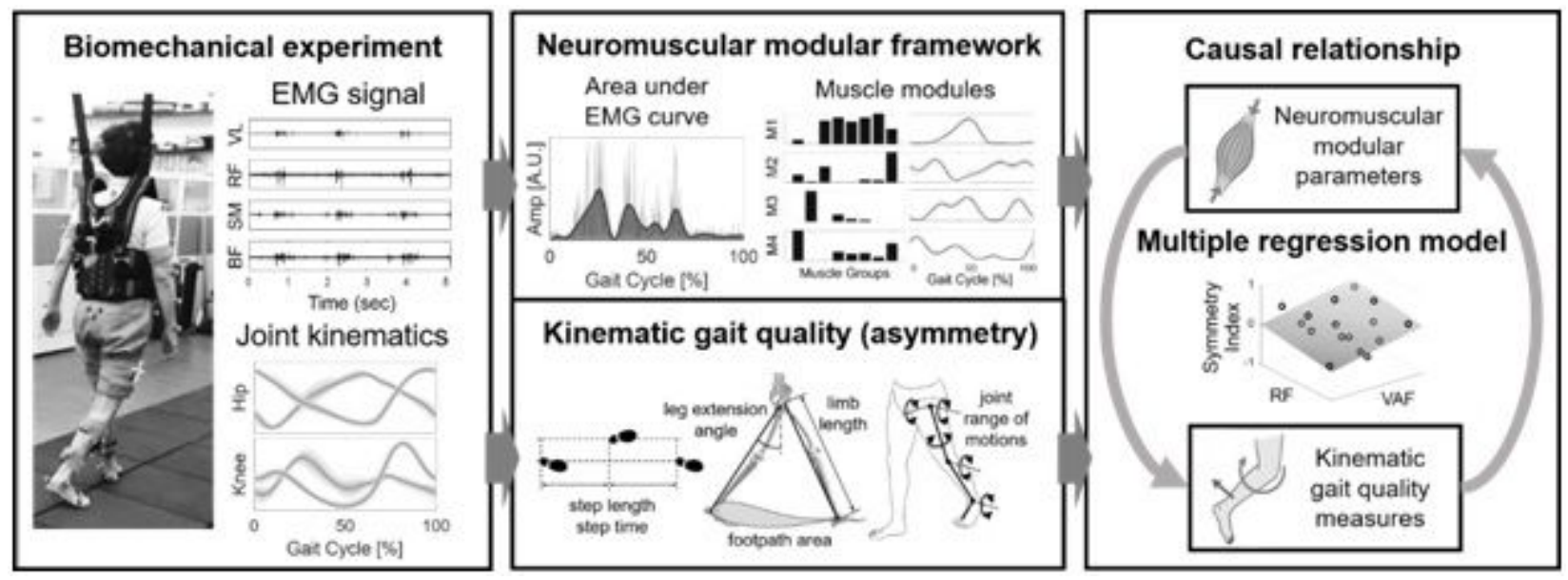

Figure 1

Overview of the study.
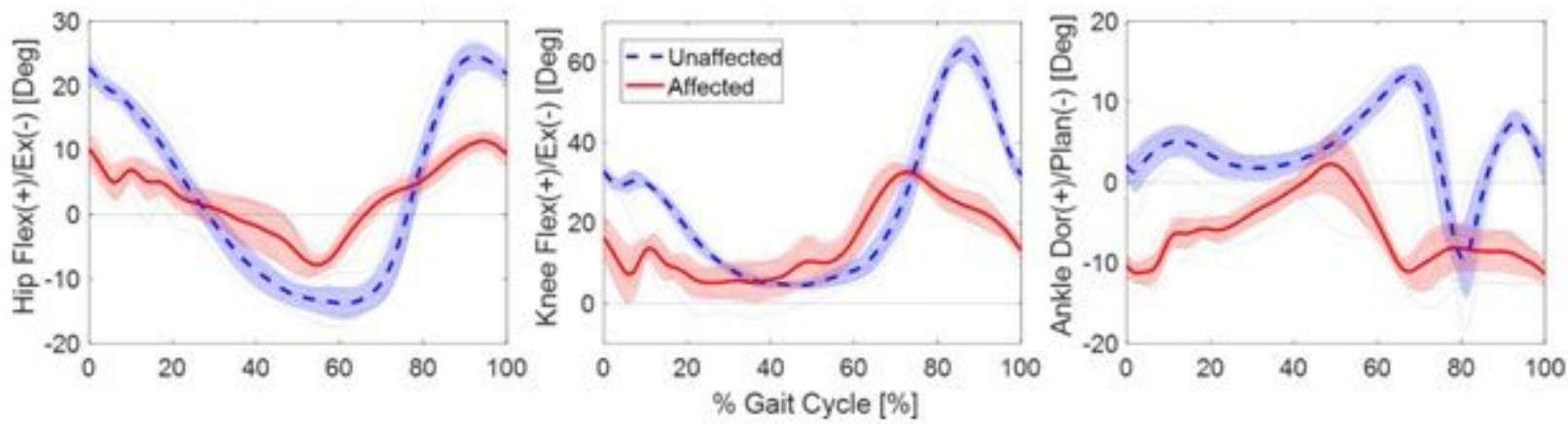

Figure 2

Example joint angle trajectories of a single gait cycle (heel strike to heel strike) in sagittal plane, hip flexion/extension (left), knee flexion/extension (middle), and ankle dorsi/plantarflexion (right).

EMG at Tibialis Anterior Muscle (TA)

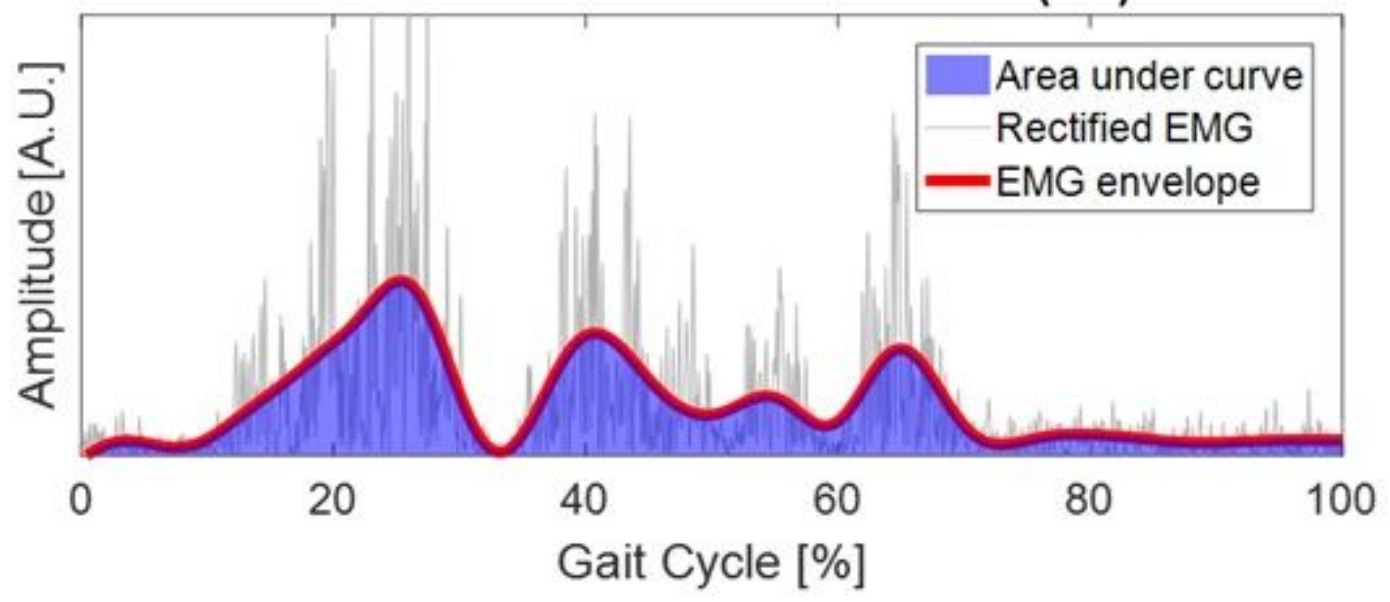

Figure 3 
Example area under the EMG envelope curve oftibialis anterior muscle from participant P1. A.U. in the $y$ axis indicates arbitrary unit.
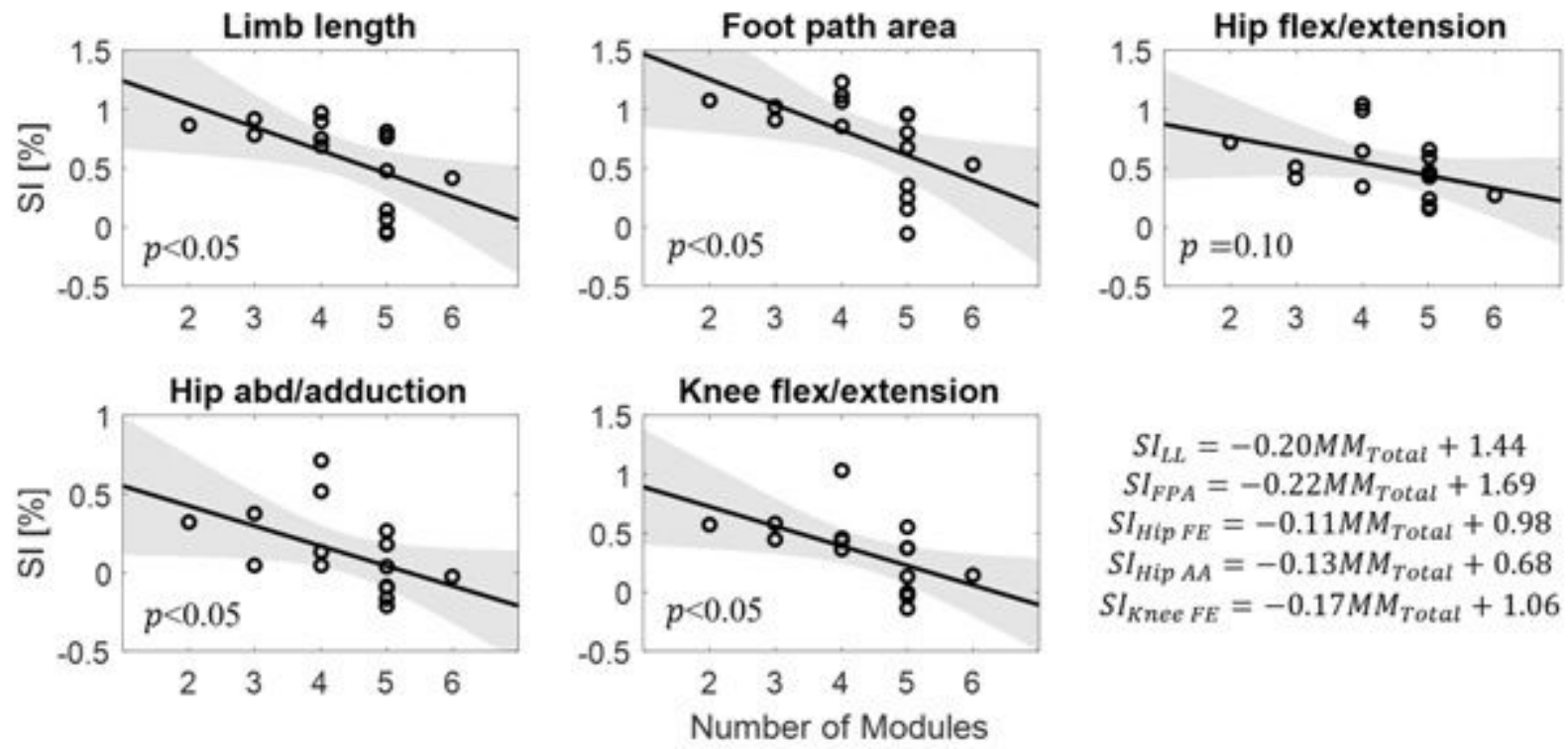

$$
\begin{gathered}
S I_{L L}=-0.20 M M_{\text {Total }}+1.44 \\
S I_{F P A}=-0.22 M M_{\text {Total }}+1.69 \\
S I_{\text {Hip FE }}=-0.11 M M_{\text {Total }}+0.98 \\
S I_{\text {Hip AA }}=-0.13 M M_{\text {Total }}+0.68 \\
S I_{\text {Knee FE }}=-0.17 M M_{\text {Total }}+1.06
\end{gathered}
$$

Figure 4

Symmetry index (SI) of significant gait parameters including limb length (LL), footpath area (FPA), hip flex/extension (Hip FE), hip abd/adduction (Hip AA) and knee flex/extension (Knee FE)with increased total number of muscle modules at both sides ( $\square \mathrm{MM} \mathbb{Z}_{-}$Total). Linear regression models (bottom, right) were fitted on each gait parameter. The shaded area indicates $95 \%$ confidence interval.
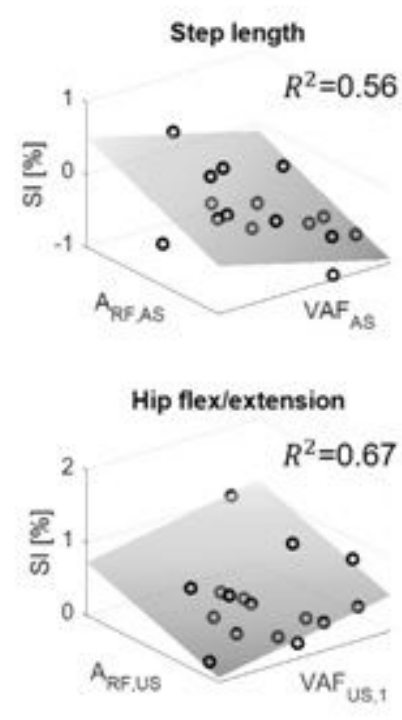

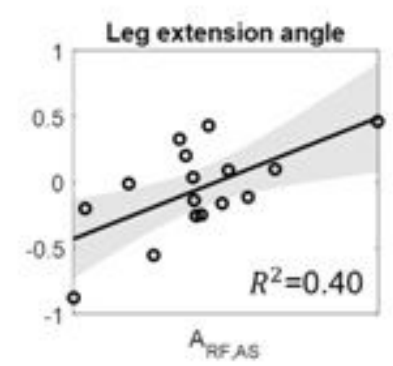

Hip abd/adduction

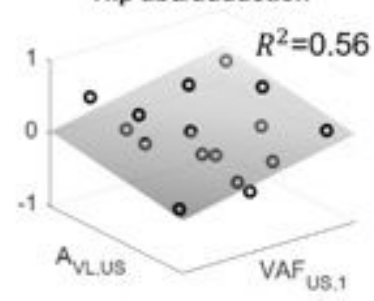

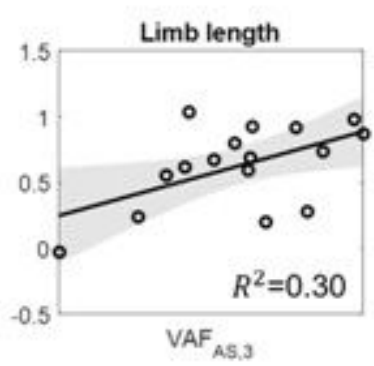

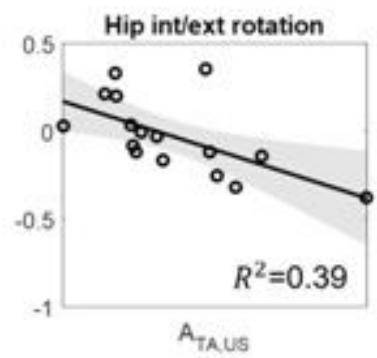

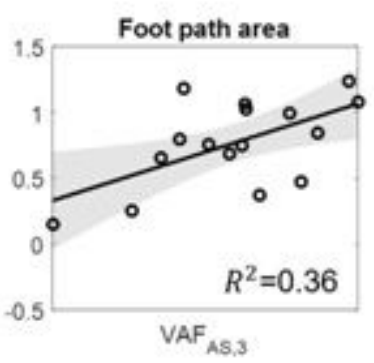

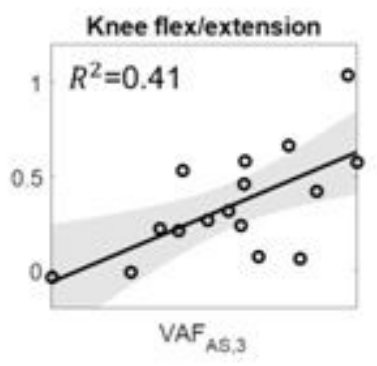

\section{Figure 5}

Regression models with selected independent variables (see Table 3)from stepwise multiple regression. Note that a plane in three-dimensional space is illustrated with two independent variables for step length, hip flex/extension and hip abd/adduction. 